\title{
Ependymoblastoma in a Japanese Black Heifer
}

\author{
Noriaki MIYOSHI ${ }^{1) *}$, Mitsuharu MATSUMOTO ${ }^{2)}$, Hiroaki KAWAGUCHI ${ }^{3)}$, Kazuyuki UCHIDA ${ }^{4)}$ and \\ Nobuhiro YASUDA ${ }^{1)}$
}

${ }^{1)}$ Departments of Veterinary Pathology, ${ }^{2)}$ Veterinary Anatomy and ${ }^{3)}$ Veterinary Laboratory Animal Science, Faculty of Agriculture, Kagoshima University, Kagoshima 890-0065 and ${ }^{4)}$ Laboratory of Veterinary Pathology, Graduate School of Agricultural and Life Sciences, The University of Tokyo, Tokyo 113-8657, Japan

(Received 19 February 2009/Accepted 10 June 2009)

ABSTRACT. An 18-month-old, Japanese Black heifer had ananastasia over a two-week period. The heifer was euthanized and necropsied. Grossly, a brown and soft mass was found in the left cerebellar hemisphere, which originated from the fourth ventricle in the pons. Multiple papillary masses were also found on the wall of the fourth ventricle. There was massive dilatation of the cerebral aqueduct, and also in the third and lateral ventricles. Histologically, the neoplastic cells formed numerous ependymoblastic rosettes and perivascular pseudorosettes. In the fourth ventricle, the normal ependymal cells were replaced by papillary proliferation of the neoplastic cells. Ultrastructually, the neoplastic cells had a feature differentiating to the ependymal cell showing a few basal bodies in the cytoplasm and connecting to adjacent cells with junctional complexes. We diagnosed the present case as ependymoblastoma with its origins in the fourth ventricle.

KEY WORDS: brain tumor, cattle, central nervous system, ependymoblastoma.

Ependymoblastoma is a rare primitive neuroectodermal tumor (PNET) exhibiting primitive ependymal differentiation in human infants and children. This neoplasm is characterized by uniform neuroepithelial cells, ependymoblastic rosettes that are different from the mature ependymal rosettes, and perivascular pseudorosettes within fields of undifferentiated cells $[1,2,6]$. This tumor is quite rare in animals with only one reported case found in a dairy calf [5]. In this report, we describe the morphologic characteristics and immunohistochemical staining pattern in the second case of ependymoblastoma in cattle.

An 18-month-old, five-month pregnant, Japanese black heifer had been anorexia and ataxia for two months. Moreover, ananastasia was observed over a two-week period. As the prognosis was poor, the animal was euthanized. Postmortem examination was performed, and tissue samples were obtained, fixed in $10 \%$ buffered formalin, and routinely processed for histopathological examination. Additionally, representative sections were applied immunohistochemical staining by the avidin-biotin-peroxidase complex method (Vectastain Elite kit, Vector Laboratories, Burlingame, CA, U.S.A.) using primary antibodies against keratin, S-100 protein, glial fibrillary acidic protein (GFAP), vimentin (Dako Corp., Kyoto, Japan), neuron-specific enolase (NSE), and neurofilament (Nichirei Corp., Tokyo, Japan). Formalin-fixed tissues were also processed for electron microscopy.

Grossly, a brown and soft mass was in the left cerebellar hemisphere with its origins in the fourth ventricle and the pons. The left cerebellum region had was compressed by

\footnotetext{
* Correspondence to: Miyoshi, N., Department of Veterinary

Pathology, Faculty of Agriculture, Kagoshima University, 1-21-

24, Korimoto, Kagoshima 890-0065, Japan.

e-mail: miyoshi@agri.kagoshima-u.ac.jp
}

the tumor mass (Fig. 1). Multiple papillary masses were found on the surfaced the fourth ventricle and pons. There was also massive dilatation of the cerebral aqueduct, and of the third and lateral ventricles. Other gross findings included purulent pneumonia and light interstitial nephritis.

Histologically, there were numerous ependymoblastic rosettes with multiple cell layers and mitotic figures within fields of undifferentiated cells (Fig. 2). These rosettes had a central lumen delimited by a distinct limiting membrane and contained a luminal nuclei-free zone. The neoplastic cells were poorly differentiated, small, and oval or spindleshaped. The nuclei were round and hyperchromatic with evenly dispersed chromatin. Mitotic figures were numerous. The neoplastic cells also formed many perivascular

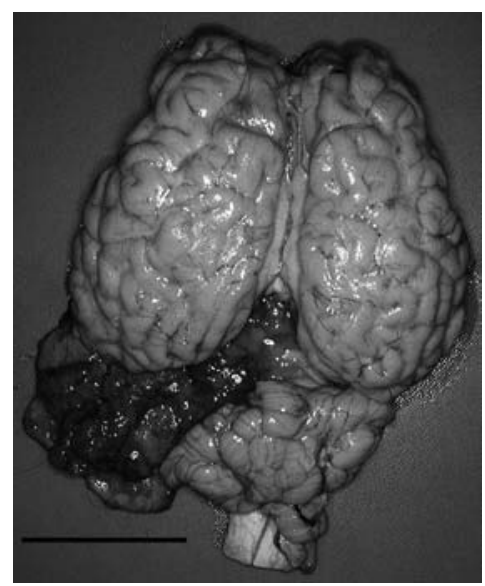

Fig. 1. Brain, dorsal view. A fragile mass is found on the left cerebellar hemisphere that exerts pressure on the cerebellar tissue. Bar $=5 \mathrm{~cm}$. 


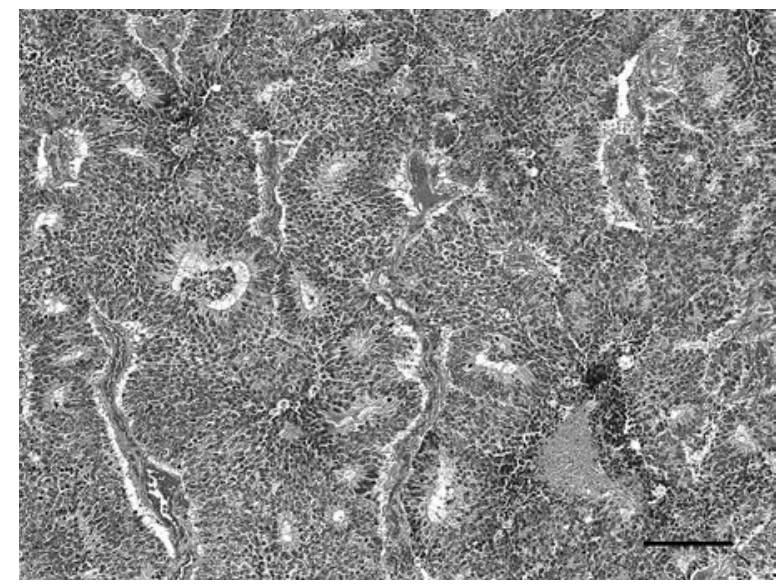

Fig. 2. Ependymoblastoma in the left cerebellum region. The neoplastic cells form numerous ependymoblastic rosettes with multiple cell layers. HE. Bar $=100 \mu \mathrm{m}$.

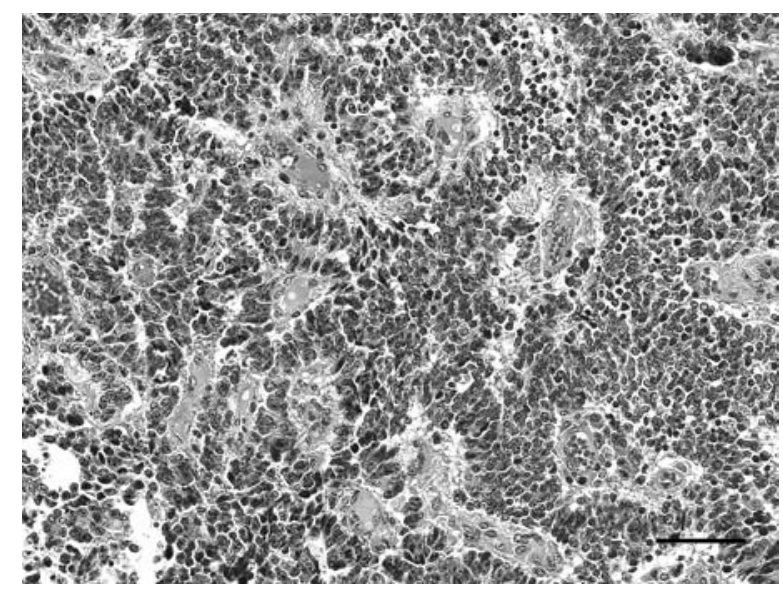

Fig. 3. Ependymoblastoma in the left cerebellum region. The neoplastic cells form perivascular pseudorosettes. HE. Bar=50 $\mu \mathrm{m}$.

pseudorosettes in the areas with more cellularity (Fig. 3). The papillary mass in the fourth ventricle, which was composed of neoplastic cells that formed many perivascular pseudorosettes, replaced normal ependymal cell layer in the pons. The neoplastic cells infiltrated into the leptomeninges, and covered the cerebellum continuously from the fourth ventricle in the pons. The cells also partially invaded into the cerebellar cortex. Large areas of necrosis, edema and hemorrhage were present.

Immunohistochemically, the neoplastic cells were negative for vimentin, keratin, S-100 protein, GFAP, NSE, and neurofilament. Ultrastructually, the neoplastic cells appeared poorly differentiated and had relatively uniform and large nuclei with a high nucleo-cytoplasmic ratio. In the periluminal portion of the ependymoblastic rosette, the neoplastic cells had a feature differentiating to the ependymal cell, showing large amounts of cytoplasm with a few basal bodies and organelles, and connecting to adjacent cells by

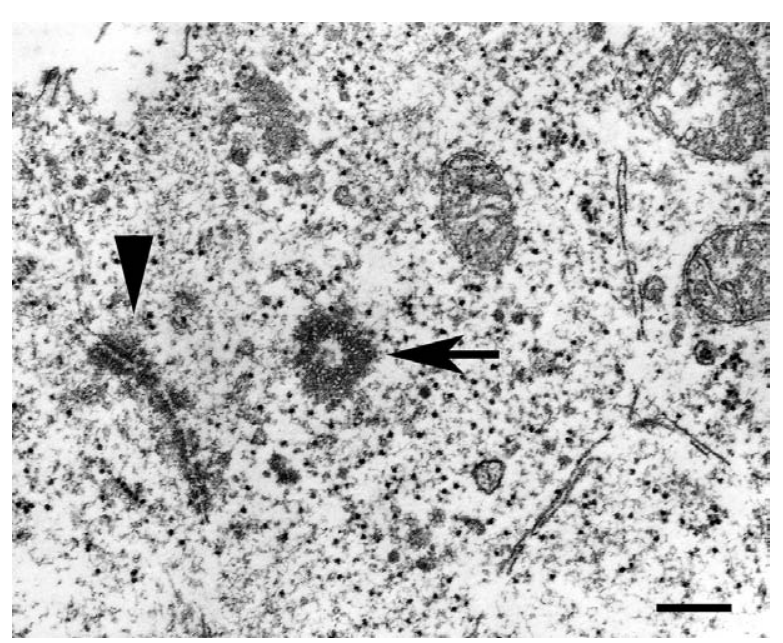

Fig. 4. Electron micrograph of the tumor cells of a formalinfixed specimen, showing a basal body (arrow) and junctional complexes (arrow-head). Bar=200 nm.

long or short junctional complexes (Fig. 4). Basement membrane was not seen.

In the present case, based on the results of the postmortem, histopathological, immunohistochemical and ultrastructural examination, the tumor was diagnosed as ependymoblastoma arising from the fourth ventricle with expansive proliferation along the leptomeninges over the cerebellum. The neoplastic cells were primitive neuroepithelial cells with ependymal differentiation, and formed ependymoblastic rosettes similar to a primitive neural tube. Additionally, the morphologic characteristics of the present case are comparable to the previous report of ependymoblastoma in a dairy calf [5]. Kleinert [3] reported that two of six human ependymoblastoma cases showed only a few GFAP- and vimentin-positive cells and suggested that tumors negative for the neuronal markers as well as for GFAP, S-100 protein, myoglobin, and desmin could be classified as PNET-NOS (not otherwise specified). Therefore, in the present case, the tumor is likely to be included in PNET-NOS immunohistochemically.

Other differential diagnoses could include anaplastic ependymoma, medulloblastoma, and medulloepithelioma. In anaplastic ependymoma, there are prominent perivascular pseudorosettes but rare in ependymal rosettes $[1,2]$. Unlike with ependymoblastoma, embryonal components and ependymoblastic rosettes are rarely seen in anaplastic ependymoma $[1,2]$. Medulloblastoma, the most common primary central nervous system neoplasm of young cattle (less than one year of age with one published exception), is usually located in the cerebellum, and characterized by Homer-Wright rosettes that lack a central lumen [4]. However, unlike the present case, ependymoblastic rosettes are rare in medulloblastoma $[1,2,4,6]$. Medulloepithelioma is characterized by papillary, tubular or trabecular arrangements of neoplastic neuroepithelium mimicking the embryonic neural tube $[1,2]$. The nuclei in medulloepithelioma 
cells more fully occupy the entire thickness of the epithelium, but, unlike ependymoblastoma, lack the nuclei-free zone of the ependymoblastic rosettes $[1,2]$.

ACKNOWLEDGMENTS. We are grateful to Mr. Y. Aitani and Mr. T. Hifumi for their valuable technical assistance.

\section{REFERENCES}

1. Becker, L. E. and Cruz-Sanchez, F. F. 2000. Ependymoblastoma. pp. 127-128. In: Pathology and Genetics of Tumors of the Nervous System, World Health Organization Classification of Tumors (Kleihues, P. and Cavenee, W. K. eds.), IARC Press, Lyon, France.

2. Burger, P. C. and Scheithauer, B. W. 1994. Embryonal tumors. pp. 193-225. In: Tumors of the Central Nervous System, Armed Forces Institute of Pathology, Wash., D.C., U.S.A.

3. Kleinert, R. 1991. Immunohistochemical characterization of primitive neuroectodermal tumors and their possible relationship to the stepwise ontogenetic development of the central nervous system. 2. Tumor studies. Acta Neuropathol. 82: 508515.

4. Sammim, D., Doherty, M. and Bassett, H. 1996. A case of cerebellar medulloblastoma in an eighteen-month-old steer. Irish Vet. J. 49: 664-665.

5. Saunders, G. K. 1984. Ependymoblastoma in a dairy calf. Vet. Pathol. 21: 528-529.

6. Summers, B. A., Cummings, J. F. and de Lahunta, A. 1995. Tumors of the central nervous system. pp. 351-401. In: Veterinary Neuropathology, Mosby-Year Book, St. Louis, U.S.A. 